\section{IS NETWORKING BEYOND BORDERS POSSIBLE? EVALUATION OF CONNECT.ED: A SECTOR-WIDE NETWORKING STRATEGY}

Venessa Wells, Rachel Meade, Roisin Sweeney. Injury Control Council of Western Australia, Australia

10.1136/injuryprev-2016-042156.676

Background Networking is an essential element of practice and developing partnerships, which often requires a significant investment of time, resources and skills.

Description of the problem Research revealed that injury prevention practitioners face complex challenges that can hinder their capacity to network and build partnerships. Challenges like restrictive budgets, heavy workloads, confidence in networking and sector diversity, which are amplified when geographically isolated, working in regional and remote Australia.

CONNECT.ed is an innovative network for people with an interest in preventing injury and creating safer communities. Designed to overcome some networking challenges, this paper describes the implementation and evaluation findings of CONNECT.ed.

Results CONNECT.ed's aim was to support regionally-based Western Australian injury prevention practitioners enhance their partnership building skills by increasing access to networking opportunities. Since inception in 2015 , it is now a network without boarders, spanning multiple countries and engaging practitioners from a diverse array of safety topics.

CONNECT.ed pairs practitioners randomly across the network, inviting them to participate in a short mutually beneficial conversation with a peer without a set agenda.

Differentiating itself from other networking and mentoring programs, CONNECT.ed is built upon the concept that all practitioners, regardless of position, experience or background, have something to share and learn from others.

CONNECT.ed utilises technology for connecting participants, removing status, providing legitimacy and permission for people to talk with others they would not normally meet.

Conclusion CONNECT.ed's format challenges the convention that networking can only occur face to face and is time and resource intensive. CONNECT.ed is playing an active role as an enabling structure in the injury prevention sector and provides an innovative opportunity to network without borders.

\section{ASSOCIATION BETWEEN HEALTH INSURANCE AND LONG-TERM INJURY-RELATED DISABILITY IN VIETNAM}

${ }^{1}$ Abdulgafoor M Bachani, ${ }^{1}$ Xiaoge Julia Zhang, ${ }^{1}$ Rantimi Oluwasegun, ${ }^{2}$ Cuong Viet Pham,

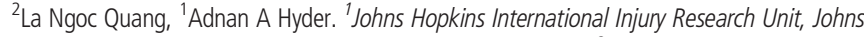
Hopkins University Bloomberg School of Public Health, USA; ${ }^{2}$ Center for Injury Policy and Prevention Research, Hanoi School of Public Health, Vietnam

\subsection{6/injuryprev-2016-042156.677}

Background Health insurance is an important social protection mechanism to insure vulnerable populations against health shocks caused by injury. This study aims to examine the association between health insurance and long-term injury-related disability in Vietnam.

Methods We are conducting a prospective cohort study of 1200 moderate to severe injury patients recruited from a public hospital in Ninh Binh, Vietnam. We administer a baseline and four follow-up surveys (at 1, 2, 4, 12 months after discharge) to participants about health insurance, socio-demographic and injury characteristics, and self-assessed disability (using WHO disability assessment schedule 2.0 (WHODAS). The outcome measure is WHODAS score.

Results We have recruited 1094 injury patients at baseline; 611 (44\%) had health insurance and 483 (56\%) did not. Insured subjects were on average 48 years in age, $64 \%$ male, and $40 \%$ farmers; while uninsured patients were on average 37 years in age, $77 \%$ male, and $>50 \%$ were farmers. Road traffic and falls were the top two causes of injury for the insured $(59 \%$ and $27 \%$ respectively) and uninsured (71\% and 14\%, respectively). Insured patients had significantly higher WHODAS global average score, 1.86 (SD: 4.8), on a scale of $0-48$ (0 for no functional limitation and 48 for extreme limitation) than the uninsured patients $(0.49$, $\mathrm{SD}: 2.1)(\mathrm{p}<0.001)$. We will use propensity score matching methods with mixed-effects models to examine the association between insurance status and average WHODAS score over time. Conclusions Health insurance may be associated with health care access prior to injury, and may influence the medical care and rehabilitation services throughout the functional recovery. This study will provide empirical evidence on how health insurance status is associated with disability related to injury over time in Vietnam.

\section{INTEGRATING INJURY PREVENTION AND CARE INTO THE BACHELOR OF NURSING SCIENCE PROGRAM OF PRABOROMAJCHANOK INSTITUTE, THAILAND}

${ }^{1}$ Sukjai Charoensuk, ${ }^{2}$ Kamolrat Turner, ${ }^{3}$ Panarut Wisawatapnimit,
'Sirikul Karuncharernpanit. ' ${ }^{2}$ Boromarajonani College of Nursing, Chakriraj; ${ }^{2}$ Boromarajonani
College of Nursing, Chon Buri; ${ }^{3}$ Boromarajonani College of Nursing, Bangkok, Thailand

\subsection{6/injuryprev-2016-042156.678}

Background The training injury prevention and care for nursing students is important as they will graduate with knowledge and skills to prevent injury and provide optimal care for people suffering from injuries in the future. This study was conducted to develop the injury prevention and care courses integrating into the bachelor of nursing science program and to train nursing instructors to be able to implement the courses.

Methods The study was conducted in 2 phases; Phase 1 developing the injury prevention and care courses, and phase 2 training nursing instructors. Participants in the first phase composed of 6 nursing instructors and 30 directors of nursing colleges. Selected experienced nursing instructors and researchers developed the injury prevention and care courses. The meeting among 30 directors of nursing colleges was set to distribute a policy of implementing the integrating injury prevention and care courses in 30 nursing colleges. In Phase 2, 51 nursing instructors from 28 nursing colleges were recruited to attend a four-day workshop. Knowledge and attitude on injury prevention and care of nursing instructors, as well as satisfaction were assessed using questionnaires developed by researchers. The scores of knowledge and attitude were compared between before and after training using paired $t$-test.

Results Four modules of injury prevention and care were developed to flexibly integrate into the bachelor of nursing science program. After the workshop, mean scores of knowledge and attitude of the nursing instructors were significantly higher than those before training $(p<0.01)$. The participants also reported their satisfaction in a high level (Mean $=4.58$, S.D. $=1.10$ ). Conclusions The four-module of integrating injury prevention and care into the bachelor of nursing science program has been 